\title{
lon-dominated plasma and the origin of jets in quasars
}

\author{
A. Hujeirat ${ }^{1}$, M. Camenzind ${ }^{2}$, and M. Livio $^{3}$ \\ 1 Max-Planck-Institut für Astronomie, 69117 Heidelberg, Germany \\ 2 Landessternwarte-Koenigstuhl, 69117 Heidelberg, Germany \\ 3 Space Telescope Science Institute, 3700 San Martin Drive, Baltimore, MD 21218, USA
}

Received 22 July 2002 / Accepted 3 September 2002

\begin{abstract}
Low cooling plasmas associated with large kinetic energies are likely to be the origin of the kpc-extended and well collimated extra-galactic jets.

It is proposed that jets are launched from a layer, governed by a highly diffusive, super-Keplerian rotating and thermally dominated by virial-hot and magnetized ion-plasma. The launching layer is located between the accretion disk and the corona surrounding the nucleus. The matter in the layer is causally connected to both the disk and to the central engine. Moreover we find that coronae, in the absence of heating from below, are dynamically unstable to thermal ion-conduction, and that accretion disks become intrinsically advection-dominated.

We confirm the capability of this multi-layer model to form jets by carrying out 3D axisymmetric quasi-stationary MHD calculations with high spatial resolution, and taking into account turbulent and magnetic diffusion. The new multi-layer topology accommodates several previously proposed elements for jet-initiation, in particular the ion-torus, the magneto-centrifugal and the truncated disk - advective tori models.
\end{abstract}

Key words. galaxies: jets, accretion disks - black holes, methods: numerical, plasma, MHD

\section{Introduction}

Jets have been observed in many systems including active galaxies, X-ray binaries, black holes X-ray transients, supersoft X-ray sources and young stellar objects (Königl 1997; Livio 1999; Mirabel 2001). Each of these systems is considered to contain an accretion disk, while jet-speeds have been verified to be of the order of the escape velocity at the vicinity of the central object (Mirabel 1999; Livio 1999 and the references therein). Recent observations of the M 87 galaxy reveal a significant jet-collimation already at 100 gravitational radii from the central engine, and that jet-launching should occur close to the last stable orbit (Biretta et al. 2002).

Several scenarios have been suggested to uncover the mechanisms underlying jet-initiations and their connection to accretion disks (Pudritz \& Norman 1986). In most of these models magnetic fields (-MFs) are considered to play the major role in powering and collimating jets (e.g., the magnetocentrifugal acceleration model of Blandford \& Payne 1982, the ion-torus model of Rees et al. 1982, X-point model of Shu et al. 1994, ADAF and ADIOS models of Narayan \& Yi 1995 and Blandford \& Begelman 1999).

Previous radiative hydrodynamical studies without magnetic fields have confirmed the formation of a transition layer (-TL) between the disk and the corona, governed by

Send offprint requests to: A. Hujeirat, e-mail: hujeirat@mpia-hd.mpg.de thermally-induced outflows (Hujeirat \& Camenzind 2000). The aim of this paper is to show that incorporating large scale magnetic fields (-MFs) manifests such formation and dramatically strengthen the dynamic of the in- and out-flows. Moreover, the TL is shown to be an optimal runaway region where highly energetic ion-jets start off. The back reaction of jet-flows on the structure of the disk and on the corona surrounding the nucleus is investigated also.

The study is based on self-consistent 3D axi-symmetric quasi-stationary MHD calculations, taking into account magnetic and hydro-turbulent diffusion, and adopting the twotemperature description (Shapiro et al. 1976). This adaptation is fundamental as 1) the dynamical time scale around the last stable orbit may become shorter than the Coulomb-coupling time. Therefore turbulent dissipation, adiabatic or shock compression preferentially heat up the ions rather than electrons $\left(T_{\mathrm{i}} \propto \rho_{\mathrm{i}}^{2 / 3}\right.$, while $\left.T_{\mathrm{e}} \propto \rho_{\mathrm{i}}^{1 / 3}\right)$. 2) Taking into account that ions radiate inefficiently, having virial-heated ions in the vicinity of the last stable orbit is essential for the total energy-budget of large scale jets.

\section{Formation of the super-Keplerian layer}

Angular momentum in standard disks (Shakura \& Sunyaev 1973, hereforth SS-disks) is transported outwards mainly via small scale magneto-hydrodynamical turbulence. Magnetic fields (-MFs) however were assumed to be weak, and remain 


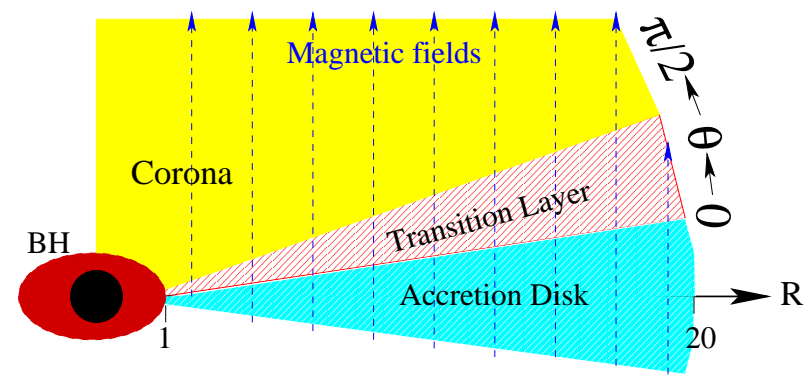

Fig. 1. The model consists of $10^{8} M_{\odot}$ Schwarzschild BH at the center (its gravity is described in terms of the quasi-Newtonian potential of Paczynski \& Wiita 1980), and an SS-disk (blue color, extending from $r=1$ to $r=20$ in units of the radius of the last stable orbit i.e., in $3 \times R_{\text {Schwarzschild }}$, thickness $H_{\mathrm{d}}=0.1 r$, an accretion rate of $\dot{\mathcal{M}}=0.01 \times \dot{\mathcal{M}}_{\mathrm{Edd}}$, and a central disk temperature of $T=10^{-3} T_{\text {virial }}$ at the outer radius). The ion-temperature $T_{\mathrm{i}}$ is set to be equal to the electron temperature $T_{\mathrm{e}}$ initially.) The low-density hot corona ( $T=T_{\text {virial }}$, and density $\rho(t=0, r, \theta)=10^{-4} \rho(t=0, r, \theta=0)$ is set to envelope the disk. A large scale magnetic field is set to thread the disk and the overlying corona (blue lines, $\beta=P_{\mathrm{mag}} / P_{\mathrm{gas}}=1 / 4$ at the outer radius, where $P_{\text {gas }}$ is the ion-pressure, $P_{\text {mag }}=B \cdot B / 8 \pi$ is the magnetic pressure, and $B$ is the magnetic field whose components are $\left(B_{\mathrm{P}}, B_{\mathrm{T}}\right)=\left(B 1, B 2, B_{\mathrm{T}}\right)$.) The low-density hot corona $\left(T=T_{\text {virial }}\right.$, and density $\rho(t=0, r, \theta)=10^{-4} \rho(t=0, r, \theta=0)$ is set to envelope the disk. A large scale magnetic field is set to thread the disk and the overlying corona (blue lines, $\beta=P_{\text {mag }} / P_{\text {gas }}=1 / 4$ at the outer radius, where $P_{\text {gas }}$ is the ion-pressure, $P_{\text {mag }}=B \cdot B / 8 \pi$ is the magnetic pressure, and $B$ is the magnetic field whose components are $\left(B_{\mathrm{P}}, B_{\mathrm{T}}\right)=\left(B 1, B 2, B_{\mathrm{T}}\right)$.) The numerical procedure is based on using the implicit solver IRMHD3 to search steady-state solution for the 3D axi-symmetric two-temperature diffusive MHD equations in spherical geometry (for further clarifications about the equations and the numerical method see Hujeirat \& Rannacher 2001; Hujeirat \& Camenzind 2000). The ion-pressure is used to describe the turbulent viscosity: $v_{\text {turb }}=\alpha P_{\text {gas }} / \Omega$, where $\alpha$ is the usual viscosity coefficient, and $\Omega$ is the angular frequency. The magnetic diffusivity is taken to be equal to $v_{\text {turb }} .250 \times 80$ strongly stretched finite volume cells in the radial and vertical direction have been used. Normal symmetry and antisymmetry boundary conditions have been imposed along the equator and the rotation axis. Extrapolation has been adopted to fix downstream values at the inner boundary. Non-dimensional formulation is adopted, using the reference scaling variables: $\tilde{\rho}=2.5 \times 10^{-12} \mathrm{~g} \mathrm{~cm}^{-3}$, $\tilde{T}=5 \times 10^{7} \mathrm{~K}, \tilde{U}=\tilde{V}_{\mathrm{S}}=\gamma \mathcal{R}_{\mathrm{g}} \tilde{T} / \mu_{\mathrm{i}},\left(\mu_{\mathrm{i}}=1.23\right) . \tilde{B}=\tilde{V}_{\mathrm{S}} \sqrt{4 \pi \tilde{\rho}}$. The location of the transition layer (-TL), where the ion-dominated plasma is expected to rotate super-Keplerian and being accelerated into jets, is shown for clarity.

so during the whole viscous evolution of the disk. Here we adopt a different view. Let $v_{\text {tot }}=v_{\mathrm{HD}}+v_{\mathrm{Mag}}=\alpha\left(P_{\text {gas }}+\pi_{\mathrm{B}}\right) / \Omega$ be a modified dynamical viscosity (see the caption of Fig. 1 for elaboration). Unlike $v_{\mathrm{HD}}$ which transports angular momentum outwards, $v_{\text {Mag }}$ transports angular momentum in the vertical direction, provided that $B_{\mathrm{P}}$ is of large scale topology. Taking into account that $v_{\mathrm{HD}}$ in SS-disks decreases inwards, a transition radius $R_{\mathrm{Tr}}$ at which result ${ }^{1}$. the time scale of angular momentum removal by MFs at $R_{\mathrm{Tr}}$ is of the same order as that

1 If $v_{\mathrm{Mag}}$ is too small, then magnetic fields are frozen-in to the gas approximately. In this case $B \sim \rho^{2 / 3}$, and $B$ must increase inwards, irrespectively, whether the disk is standard or advection-dominated. by the turbulent viscosity. Hence

$\frac{\tau_{\text {rem }}}{\tau_{\text {tur }}} \simeq \alpha\left(\frac{H}{r}\right)\left(\frac{V_{\mathrm{s}}}{V_{\mathrm{A}}}\right)^{2} \simeq 1$,

where $V_{\mathrm{s}}, V_{\mathrm{A}}$ are the sound and transverse Alfven speeds $\left(V_{\mathrm{A}}=\sqrt{B_{\mathrm{P}} B_{\mathrm{T}} / 4 \pi \rho}\right)$, respectively. Taking into account that $H / r \geq \alpha$, we end up with $V_{\mathrm{A}} \geq \alpha V_{\mathrm{s}}$. The last optimistic inequality applies everywhere in the disk, irrespective whether turbulence is mediated by $\pi_{\mathrm{B}}$ or not. Therefore, vertical transport of angular momentum via MFs is at least as efficient as $\alpha$-viscosity. This is even more justified by the fact that Balbus-Hawley instability in Keplerian-disks amplifies initially weak fields to considerably large values, but remain still below equipartition (Hawley et al. 1996). Imposing appropriate boundary conditions, and carrying the MHD-Box calculations with high spatial resolution, MFs could be amplified up to equipartition (Ziegler 2002), yielding thereby $\tau_{\text {rem }}<\tau_{\text {tur }}$. Furthermore, the dynamo-action model proposed by Tout \& Pringle (1992), if applied, would make $\tau_{\text {rem }}$ even shorter.

To be noted here that when taking a more realistic density and temperature stratification in global 3D MHD disk-calculations, vertical transport of angular momentum is inevitable (Arlt 2002).

Efficient vertical transport of angular momentum rises the following important issues: 1) Turbulence in the disk need not be dissipated, or it might be even suppressed by the amplified $B_{\mathrm{P}}$. This allows accretion to evolve without necessarily emitting the bulk of the their potential energy as radiation, and gives rise to energy re-distribution. 2) Accretion flows may not proceed as slowly as in SS-disk, but they may turn into advection-dominated ${ }^{2}$. This occurs because the time scale of angular momentum removal from the disk scales as:

$\tau_{\text {rem }} \sim \rho V_{\mathrm{T}} H / B_{\mathrm{P}} B_{\mathrm{T}} \sim r^{3 / 2}$,

where $V_{\mathrm{T}}(=r \cos \theta \Omega)$ is the angular velocity (Fig. 1). This indicates that angular momentum removal is more efficient at smaller radii.

To maintain dynamical stability, angular momentum removal from the disk should be compensated by rapid advection from larger radii, i.e., $\tau_{\text {adv }}=\tau_{\text {rem. }}$. Further, rapid and steady generation of $B_{\mathrm{T}}$ in the disk yields $B_{\mathrm{P}} / B_{\mathrm{T}}=H_{\mathrm{d}} / r$. The later two conditions imply that the radial velocity $U_{\mathrm{r}} \sim V_{\mathrm{A}}$, which means that the stronger the MF threading the disk, the more advection-dominated it becomes, and therefore the faster is the establishment of the super-Keplerian layer.

Based on the present calculations (see the caption of Figs. 1 and 2-5), it is found that: 1) Angular velocity in the transition layer (TL) adopts approximately the profile $\Omega \sim r^{-5 / 4}$. 2) Energy dissipation is injected primarily into the ions that cool predominantly through fast outflows. 3) The generated toroidal magnetic field is quenched by a magnetic diffusion (reconnection) and fast outflows. The width of the TL is predominantly determined through the transverse variation of the ion-pressure across the jet, i.e., $H_{\mathrm{W}}=P_{\mathrm{i}} / \nabla P_{\mathrm{i}} \approx 0.2 r$, and

2 The flow is said to be advection-dominated if transport via fluidmotion dominates viscous re-distribution. 
so strongly dependent on whether the flow is a one- or twotemperature plasma. In the steady-state case, this implies:

$\rho \sim r^{-7 / 4}, T_{\mathrm{i}} \sim r^{-1 / 2}, U_{\mathrm{r}} \sim r^{-1 / 4}, B_{\mathrm{P}} / B_{\mathrm{T}} \sim$ const.

We note that $U_{\mathrm{r}}$ adopts a profile and attains values similar to those in the innermost part of the disk. Provided that energy exchange between the matter in the disk and in the TL is efficient, the incoming matter can easily be re-directed into outwardsoriented motions. This implies that the Bernoulli number (Be) can change sign in dissipative flows. As Fig. 5 shows, Be is everywhere negative save the TL, where it attains large positive values, so that the ion-plasma can start its kpc-journey. Worthnoting is the resulting MF topology (see Fig. 4). Apparently, the outflow is sufficiently strong to shift the MF lines outwards, while the large diffusivity prevents the formation of large electric currents along the equator. In the corona however, MFs are too weak to halt the diffusive plasma in the dynamically unstable corona against gravity, and instead, they drift with the infalling gas inwards. In the case of very weak MFs $(\beta \leq 0.1)$, our calculations indicate a considerably weak outflow. This is a consequence of the tendency of the MFs to establish a monopole like-topology, i.e, a one-dimensional MF-topology in which $B_{\theta} \longrightarrow 0$. In this case, the magnetic tension $\pi_{\mathrm{B}}$ becomes inefficient in feeding the matter in the TL with the angular momentum required for launching jets, indicating herewith that cold accretion disks alone are in-appropriate for initiating winds (Ogilvie \& Livio 2001).

Comparing the flux of matter in the wind to that in the disk, we find that $\dot{\mathcal{M}}_{\mathrm{W}} / \dot{\mathcal{M}}_{\mathrm{d}}=$ const. $\approx 1 / 20$. The angular momentum flux associated with the wind is $\dot{\mathcal{J}}_{\mathrm{W}} / \dot{\mathcal{J}}_{\mathrm{d}}=a\left(\dot{\mathcal{M}}_{\mathrm{W}} / \dot{\mathcal{M}}_{\mathrm{d}}\right) r^{1 / 4}$, where $a$ is a constant of order unity. Consequently, at $r=3 \times 10^{2} R_{\mathrm{Sch}}$, almost $25 \%$ of the total accreted angular momentum in the disk re-appears in the wind.

Why is the TL geometrically thin?

In stratified dissipative flows the density scale height is much smaller than the scale height of the angular velocity (Hujeirat $\&$ Camenzind 2000). Since the flow in TL rotates superKeplerian, Coriolis forces act to compress the disk-matter and make its density scale height even smaller. This implies that advective-disks are geometrically thin, much thinner than what ADAF-solutions predict.

On the other hand, unless there is a significant energy flux that heats up the plasma from below, as in the case of stars, heat conduction will always force the $\mathrm{BH}$-coronae to collapse dynamically. To elaborate this point, let us compare the conduction time scale with the dynamical time scale along $B_{\mathrm{P}}$-field at the last stable orbit of a SMBH:

$\frac{\tau_{\text {cond }}}{\tau_{\text {dyn }}}=\frac{r \rho U_{\mathrm{r}}}{\kappa_{0} T_{\mathrm{i}}^{5 / 2}}=4.78 \times 10^{-4} \rho_{10} T_{\mathrm{i}, 10}^{-5 / 2} \mathcal{M}_{8}$,

where $\rho_{10}, T_{\mathrm{i}, 10}$ and $\mathcal{M}_{8}$ are respectively in $10^{-10} \mathrm{~g} \mathrm{~cm}^{-3}$, $10^{10} \mathrm{~K}$ and in $10^{8} \mathcal{M}_{\odot}$ units. This is much less than unity for most reasonable values of density and temperature typical for AGN-environments. In writing Eq. (4) we have taken optimistically the upper limit $c / \sqrt{3}$ for the velocity, and
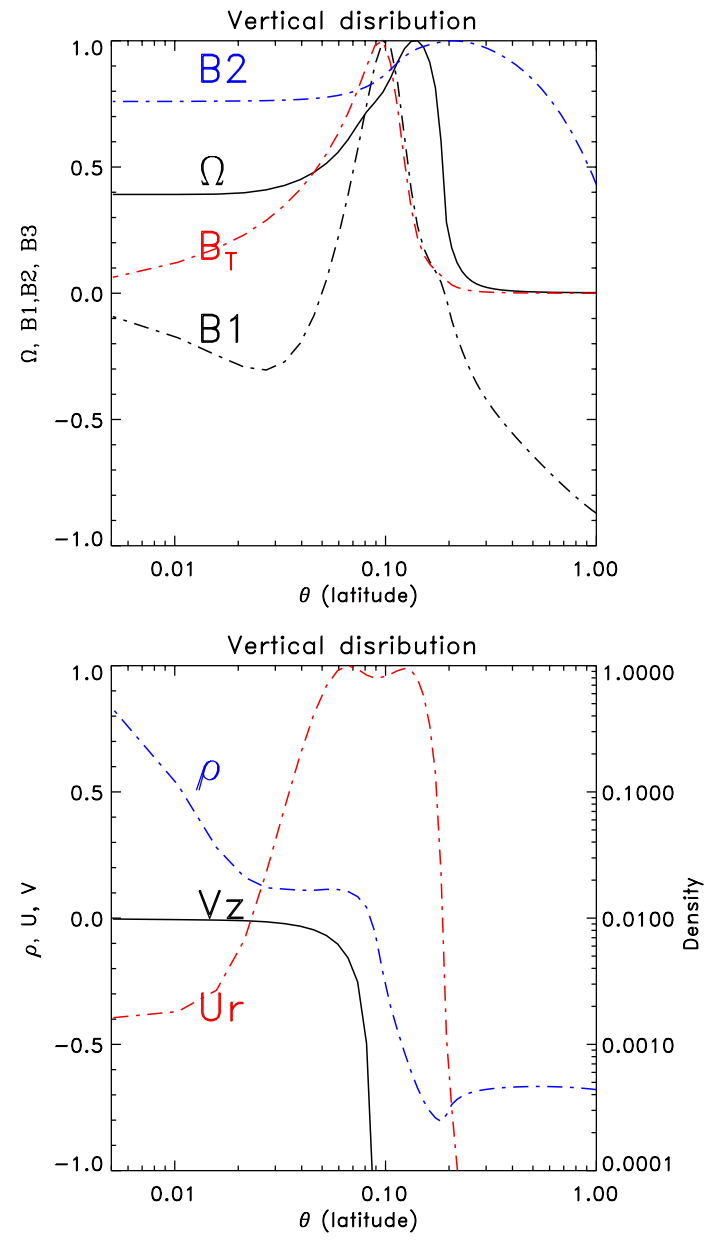

Fig. 2. The horizontal distribution of the normalized density $\rho$, angular velocity $\Omega$, radial and horizontal velocities $U_{\mathrm{r}}, V_{\mathrm{z}}$, the radial and horizontal MF-components $(B 1, B 2)=\left(B_{\mathrm{r}}, B_{\theta}\right)=B_{\mathrm{P}}$ and the toroidal MF-component $B_{\mathrm{T}}$ at $r=2.5$. Note the density plateau, the positive radial velocity (outflows), the super-Keplerian rotation and the strongly enhanced strength of the MF-components in the TL. The inwardsoriented motions in the disk (inflows) and strongly increasing density towards the equator are obvious.

set $\kappa_{0}=3.2 \times 10^{-8}$ for the ion-conduction coefficient. When modifying the conduction operator to respect causality, we obtain $\tau_{\text {cond }} / \tau_{\text {dyn }} \leq U_{\mathrm{r}} / c$, which is again smaller than unity.

This agrees with our numerical calculations which rule out the possibility of outflows along the rotation axis, and in particular not from the highly unstable polar region of the $\mathrm{BH}$, as ADAFsolutions predict.

\section{Summary}

We have presented a multi-layer model for initiating ion-jets in AGNs. The model agrees with a previous numerical study which confirmed the formation of thermally-induced outflows in the transition layer (Hujeirat \& Camenzind 2000). Here we have shown that incorporating the effects of MFs manifests their formation and dramatically strengthen their dynamics.

Three ingredients for initiating winds have been detected: 1) a highly diffusive plasma dominated by virial-hot ions, 2) large scale magnetic fields that efficiently transport angular 

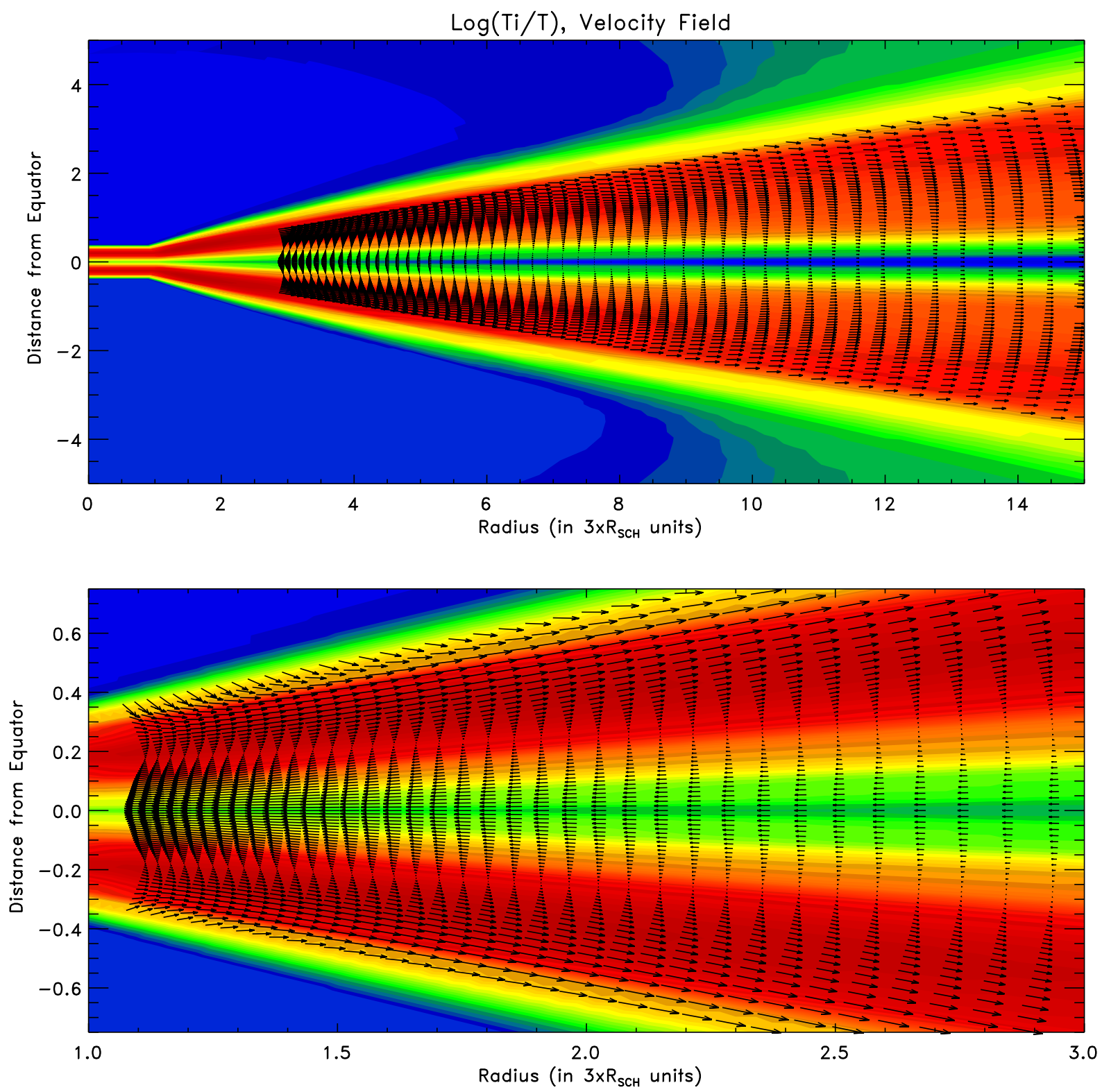

Fig. 3. The distribution of the velocity field superposed on the logarithmic-scaled ratio of the ion- to electron-temperatures (red color corresponds to high ratios and blue to low-ratios). The lower figure is a zoom-in of the flow configuration in the innermost part of the disk.

momentum from the disk into the TL, where the plasma rotates super-Keplerian, and 3) an underlying advection-dominated accretion disk.

Taking into account that the corona is dynamically unstable, adopting a large scale magnetic topology, and allowing ion-electron thermal decoupling appear to force accretion flows to undergo a global energy re-distribution: confined inflows (negative Bernoulli number) in the equatorial region and in the corona, and thermally and magneto-centrifugally-driven outflows in the TL characterized through a positive Bernoulli number. This feature may survive under different conditions: strong MFs suppress turbulence, weakening thereby the effect of the turbulent-viscosity and dominate the transport of angular momentum. On the other hand weak MFs in rotating stratified flows would be amplified via dynamo-action and reach equipartition, beyond which turbulence is again suppressed.
This interplay between MFs and turbulent-viscosity, BalbusHawley and Parker instabilities may settle into an equilibrium state, in which inflows are simultaneously associated with lowcooling out-flowing ion-plasma.

We note that in the absence of thermal conduction and adopting the one-temperature description, low-viscosity radiatively inefficient HD and MHD accretion flows become inevitably convection-dominated. Therefore, in the early phases of jet-initiation, CDAFs may play an important role in powering the jets in AGNs and microquasars (Abramowicz et al. 2002).

The multi-layer model presented here accommodates some elements of BP82. In particular, we agree with BP82 about the necessity for a super-Keplerian rotation of the plasma overlying the accretion disk. However, the plasma here is dominated by highly-diffusive and virial-hot ions; it does not require a special 


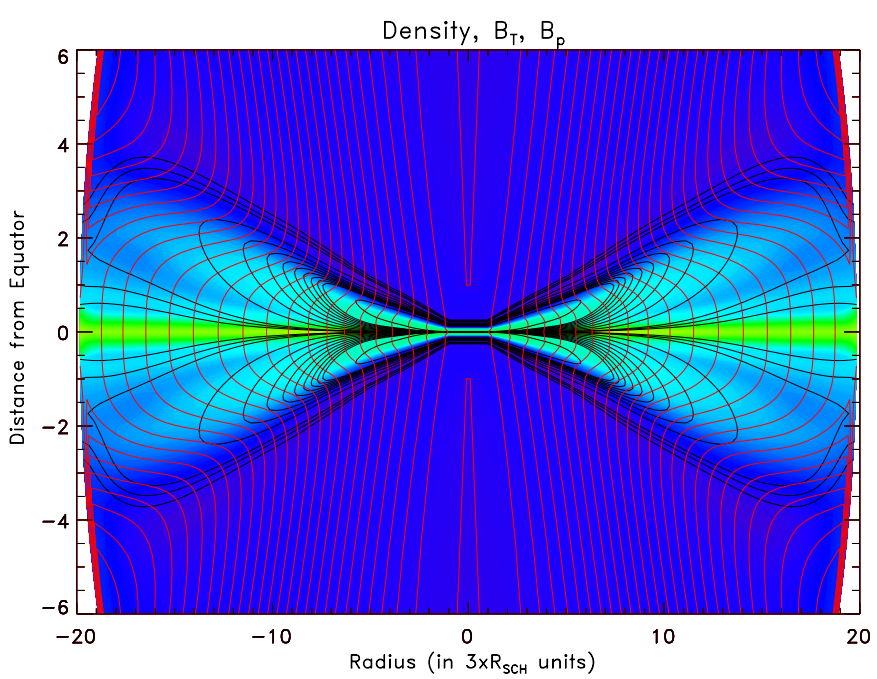

Fig. 4. 40 equally-spaced isolines of the poloidal-component $B_{\mathrm{P}}$ (red lines) and 30 equally-spaced isolines of $B_{\mathrm{T}}$ (black lines) superposed on the density-distribution (yellow color corresponds to very high density-values, blue to middle and violet to low values). $B_{\mathrm{T}}$ attains it maximum values in the innermost part of the transition layer and diminishes in the corona.

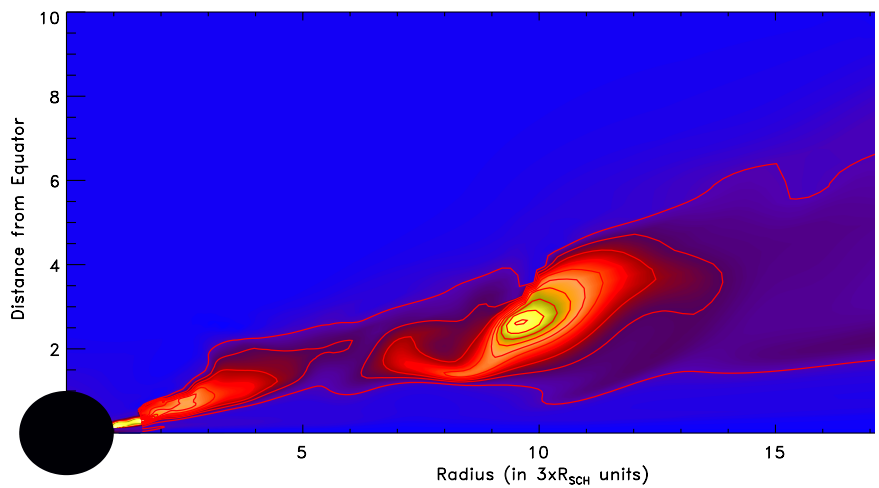

Fig. 5. A snap-shot of the distribution of the Bernoulli number in twodimensional time-dependent calculations. The decrease from large to low positive values is represented via yellow, green and red colors. The blue color corresponds to negative values. The figure shows two ejected blobs of large positive energies in the TL. Ejection starts from the innermost region of the TL and evolves non-linearly.

$B_{\mathrm{P}}$-alignment with respect to the disk-normal to enable jetlaunching, as ideal-MHD treatment requires. While our results agrees with the ion-torus model with respect to the necessity of 2T-plasma to maintain the ions hot for a significant time of their propagation-life in the ISM, no signatures for the formation of ion-supported tori have been detected (Rees et al. 1982).

Our results differ from ADAF and ADIOs in several issues, and in particular with respect to 1) the existence of a layer adjusting to the disk, where the plasma is found to rotate superKeplerian, 2) the configurations of the in- and the out-flows, 3) stability of the corona in the vicinity of the $\mathrm{BH}, 4)$ the transition from SS-disk to advection-dominated disks and 5) with respect to the essence of Bernoulli number in dissipative flows, i.e., a positive Bernoulli number is necessary but not sufficient for outflows (Abramowicz et al. 2000).

Finally, we note that since the flow in the TL is highly dissipative (strengthen thermal and rotational coupling with the central nucleus), the innermost region of the disk rotates synchronously with Kerr black holes (due to the frame dragging effect), and since $\tau_{\text {rem }}$ decreases with radius and depends inversely on $B_{\mathrm{T}}$ and $B_{\mathrm{P}}$, we think that the plasma attached to the poloidal magnetic field would be forced to deposit its angular momentum to the plasma in the TL, thereby considerably enhancing the centrifugal power and ejecting the ion-plasma into space with relativistic speeds.

\section{References}

Abramowicz, M., Lasuta, J.-P., \& Igumenshchev, I. 2000, MNRAS, 314,775

Abramowicz, M., Igumenshchev, I., Quataert, E., \& Narayan, R. 2002, ApJ, 565, 1101

Arlt, R. 2002, private communication

Balbus, S., \& Hawley, J. 1991, ApJ, 376, 214

Biretta, J., Junor, W., \& Livio, M. 2002, New Astron. Rev., 46, 239

Blandford, R., \& Payne, D. G. 1982, MNRAS, 199, 883

Blandford, R., \& Begelman, M. 1999, MNRAS, 303, L1

Hawley, F., Gammie, C., \& Balbus, S. 1996, ApJ, 464, 690

Hujeirat, A., \& Rannacher, R. 2001, New Astron. Rev., 45, 425

Hujeirat, A., \& Camenzind, M. 2000, A\&A, 362, 41

Königl, A. 1997, IAU Coll. 163, ASP, 121

Livio, M. 1999, Phys. Rep., 311, 225

Mirabel, I. F. 2001, ApSSS, 276, 153

Mirabel, I. F. 1999, ARA\&A, 37, 409

Narayan, R., \& Yi, I. 1995, ApJ, 444, 231

Ogilvie, I., \& Livio, M. 2001, ApJ, 553, 158

Pudritz, R., \& Norman, C. 1986, ApJ, 301, 571

Rees, M., Begelman, M., Blandford, R., \& Phinney, E. 1982, Nature, 295, 17

Shakura, N. I., \& Sunyaev, R. A. 1973, A\&A, 24, 337

Shapiro, S. L., Lightman, A. P., \& Eardley, D. M. 1976, ApJ, 204, 187

Shu, F., Najita, J., Ostriker, E., et al. 1994, ApJ, 429, 781

Tout, C. A., \& Pringle, J. E. 1992, MNRAS, 259, 604

Ziegler, U. 2002, private communication 